\title{
p53 mutations in classic and pleomorphic invasive lobular carcinoma of the breast
}

\author{
Cigdem Ercan • Paul J. van Diest • Bram van der Ende • \\ John Hinrichs • Peter Bult • Horst Buerger • \\ Elsken van der Wall • Patrick W. B. Derksen
}

Accepted: 31 January 2012 / Published online: 22 February 2012

(C) The Author(s) 2012. This article is published with open access at Springerlink.com

\begin{abstract}
Background $\mathrm{p} 53$ is a tumor suppressor that is frequently mutated in human cancers. Although alterations in p53 are common in breast cancer, few studies have specifically investigated TP53 mutations in the breast cancer subtype invasive lobular carcinoma (ILC). Recently reported conditional mouse models have indicated that functional p53 inactivation may play a role in ILC development and progression. Since reports on the detection of TP53 mutations in the relatively favorable classic and more aggressive pleomorphic variants of ILC (PILC) are rare and ambiguous, we performed a comprehensive analysis to determine the mutation status of TP53 in these breast cancer subtypes.

Methods To increase our understanding of p53-mediated pathways and the roles they may play in the etiology of classic ILC and PILC, we investigated TP53 mutations
\end{abstract}

C. Ercan · P. J. van Diest • B. van der Ende · J. Hinrichs •

P. W. B. Derksen $(\bowtie)$

Department of Pathology, H04.312,

University Medical Center Utrecht,

PO Box 85500, 3508 GA, Utrecht, The Netherlands

e-mail: P.W.B.Derksen@umcutrecht.nl

E. van der Wall

Division of Internal Medicine and Dermatology,

University Medical Center Utrecht,

Utrecht, Netherlands

\section{P. Bult}

Department of Pathology,

Radboud University Nijmegen Medical Centre,

Nijmegen, The Netherlands

\section{H. Buerger}

Institute of Pathology,

Paderborn, Germany and p53 accumulation in a cohort of 22 cases of classic and 19 cases of PILC by direct DNA sequencing and immunohistochemistry.

Results We observed 11 potentially pathogenic TP53 mutations, of which three were detected in classic ILC (13.6\%) and 8 in PILC (42.1\%; $p=0.04)$. While p53 protein accumulation was not significantly different between classic and pleomorphic ILC, mutations that affected structure and protein function were significantly associated with p53 protein levels.

Conclusion TP53 mutations occur more frequently in PILC than classic ILC.

Keywords Breast cancer · p53 - Mutation · Invasive lobular breast cancer $\cdot$ Classic $\cdot$ Pleomorphic

\section{Introduction}

The tumor suppressor p53 was first described in 1979 as a key cell cycle regulator. Upon cellular stress, the p53 signaling pathway turns on the expression of genes including inhibitors of cell cycle, DNA repair, and apoptosis [1,2]. Inactivating alterations in the p53 gene are commonly observed in human cancers, resulting in suppression of the regulatory functions of p53 which contributes to transformation of cells. Mutations in p53 are observed in breast cancer, however with a lower frequency $(\sim 20 \%)$ compared to other solid tumors [2]. Although it has been well established that $\mathrm{p} 53$ mutations correlate with high grade and triple negativity [3, 4], the p53 mutation spectrum across the various different histological types of breast cancer has not been well defined.

Invasive lobular cancer (ILC) accounts for approximately $15 \%$ of breast cancers [5]. Based on their molecular profile, most ILC belong to the luminal-type breast cancers. Within 
ILC, several subtypes can be discerned: 1) the classic type composed of small regular cells with frequently intracytoplasmic vacuoles, small nuclei and a typical trabecular infiltration pattern with dissociated cells or forming single files, often in targetoid patterns around uninvolved ducts and with low mitotic rate; 2) the better demarcated alveolar type exhibiting small round aggregates of 20 or more cells with typical lobular cytology; 3) the also better demarcated solid variant consisting of more solid sheets of cells with little intervening stroma, more mitoses and often some more atypia; 4) the pleomorphic variant that exhibits the growth pattern of classical lobular carcinoma throughout but with polygonal, eccentric pleomorphic nuclei and more frequent mitoses [6]. The pleomorphic variant of ILC (PILC) accounts for less than $1 \%$ of all breast carcinomas and not more than $10 \%$ of all ILC [7]. It has a poorer prognosis compared to classic ILC [5, 8]. Several studies have addressed the molecular and histological aspects of PILC as a separate entity. Immunohistochemical analyses demonstrated that PILCs frequently express estrogen (ER) and progesterone (PR) receptors and are mostly E-cadherin negative, Her2 positive and occasionally p53 positive [8-12]. Although expression of gross cystic disease fluid protein (GDCFP) has been used to facilitate differential diagnosis between classic ILC and PILC, reliability is variable [11-14]. Little is known about p53 mutation status in PILC. In view of the higher nuclear grade and poorer prognosis, one would expect a higher frequency of p53 mutation in PILC. Interestingly, conditional knock-out mouse models have indicated that functional inactivation of p53 may play a role in carcinogenesis and progression of mouse PILC $[15,16]$. In the present study, we have investigated the p53 mutation status in a series of 41 ILC cases including 22 classic and 19 pleomorphic subtypes to advance current knowledge on these variants of ILC.

\section{Materials and methods}

\subsection{Patients}

Archival material from 41 breast cancer patients with lobular carcinoma was collected from the Pathology departments of the University Medical Center Utrecht, Utrecht, The Netherlands and Radboud University Nijmegen Medical Centre, Nijmegen, The Netherlands, and the Institute of Pathology, Paderborn, Germany. ILC and PILC were identified on Hematoxylin and Eosin (H\&E) stained reference slides from formaldehyde-fixed paraffin embedded breast cancer tissue blocks of 41 cases by an experienced pathologist (PJvD), considering cases with nuclear atypia score 3 as PILC. Use of anonymous or coded left over material for scientific purposes is a part of the standard treatment contract with patients in our hospitals [17].

\subsection{DNA extraction}

After de-paraffinization of the slides by standard methods, the relevant area from each slide (as identified on corresponding H\&E stained sections) was scraped off with a scalpel and suspended in lysis buffer (Tris/HCl buffer $\mathrm{pH} 8.0$ with Tween). Then, proteinase $\mathrm{K}$ was added and the samples were incubated for $1 \mathrm{~h}$ at $56^{\circ} \mathrm{C}$. After that, samples were incubated at $95^{\circ} \mathrm{C}$ for $10 \mathrm{~min}$ to inactivate proteinase $\mathrm{K}$.

\subsection{Sequencing and mutation analysis}

For the detection of mutations, genomic DNA was amplified with primers flanking exons 4, 5, 6, 7, 8 and 9 of the TP53 gene (Table 1). The PCR conditions were set up as follows; initial denaturation at $94^{\circ} \mathrm{C}$ for $3 \mathrm{~min}, 35$ cycles at $94^{\circ} \mathrm{C}$ for $1 \mathrm{~min}$ (denaturation), $60^{\circ} \mathrm{C}$ for $30 \mathrm{~s}$ (annealing) and $72^{\circ} \mathrm{C}$ for $45 \mathrm{~s}$ (extension), followed by a final extension at $72^{\circ} \mathrm{C}$ for $5 \mathrm{~min}$. Then, PCR products were sequenced in both sense and antisense directions using the BigDye Terminator v1.1 sequencing kit on ABI 3130 (Applied Biosystems, Foster City, CA, USA) according to the manufacturer's instructions. The sequences were analyzed using Mutation Surveyor software (SoftGenetics,LLC., State College, PA, USA).

\subsection{Immunohistochemistry}

Four $\mu \mathrm{m}$ thick sections were cut from the paraffin blocks and transferred to Superfrost + slides (Menzel and Glaeser, Braunschweig, Germany). Citrate buffer was used for antigen retrieval. Immunohistochemistry was then performed

Table 1 Summary of primer sequences

\begin{tabular}{ll}
\hline Exon & Sequence \\
\hline $4.1 \mathrm{FW}$ & 5' CTG GTC CTC TGA CTG CTC 3' \\
$4.1 \mathrm{RV}$ & 5' GAC AGA AGA TGA CAG GGG 3' \\
$4.2 \mathrm{FW}$ & 5' AGC TCC CAG AAT GCC AGA G 3' \\
$4.2 \mathrm{RV}$ & 5' TGA AGT CTC ATG GAA GCC 3' \\
5.1 FW & 5' CCG TGT TCC AGT TGC TTT ATC 3' \\
5.1 RV & 5' GCT GTG ACT GCT TGT AGA TG 3' \\
$5.2 \mathrm{FWa}$ & 5' TCA ACA AGA TGT TTT GCC AAC TGG 3' \\
$5.2 \mathrm{FWb}$ & 5' ACA AGA TGT TTT GCC AAC TG 3' \\
$5.2 \mathrm{RVint}$ & 5' GAG CAA TCA GTG AGG AAT CAG 3' \\
$6 \mathrm{FW}$ & 5' TCC CCA GGC CTC TGA TTC 3' \\
$6 \mathrm{RV}$ & 5' CTG ACA ACC ACC CTT AAC C 3' \\
$7 \mathrm{FW}$ & 5' CTT GCC ACA GGT CTC CCC AA 3' \\
$7 \mathrm{RV}$ & 5' GCG GCA AGC AGA GGC TGG 3' \\
$8 \mathrm{FW}$ & 5' CCT TAC TGC CTC TTG CTT C 3' \\
$8 \mathrm{RV}$ & 5' TAA CTG CAC CCT TGG TCT C 3' \\
$9 \mathrm{FW}$ & 5' GTT ATG CCT CAG ATT CAC T 3' \\
$9 \mathrm{RV}$ & 5' TGA GTG TTA GAC TGG AAA C 3' \\
\hline
\end{tabular}


with a mouse monoclonal p53 antibody, clone BP53-12-1, $10 \mathrm{mg} / \mathrm{mL}$ stock, (MU 195-UC, BioGenex, San Ramon, CA, USA; 1:100) on an automated immunostainer (BondMaX, Leica, Bannockburn, IL). Slides were counterstained with hematoxylin, dehydrated, and cover-slipped. Throughout the immunohistochemical analysis, negative controls were obtained by omitting the primary antibody and staining of a colon cancer tissue with a verified p53 mutation was used as a positive control. Scoring of the stained nuclei was performed by an experienced pathologist (PJvD). p53 was regarded as accumulated when $\geq 5 \%$ of nuclei were stained. p53 was regarded as wild-type when approximately $1 \%$ of nuclei showed expression.

\subsection{Statistical analysis}

Chi-Squared test, or, when appropriate, Fischer's Exact test was applied to compare frequencies between groups with the SPSS 15.0 software package (IBM), regarding p-values $<0.05$ as significant.

\section{Results}

We identified the pleomorphic ILC variant using $\mathrm{H} \& \mathrm{E}$ staining, based on a classical trabecular ILC growth pattern, but with polygonal eccentric pleomorphic nuclei and more frequent mitoses (cases with nuclear atypia score 3 ). We have not observed a significant increase in HER2 expression in PILC when comparing with classical ILC in our samples (data not shown).

The rationale behind our investigation of $\mathrm{p} 53$ in classic ILC and PILC is gaining a new insight into distinctive gene alterations which give rise to these two different subtypes of ILC.

\section{$3.1 \mathrm{p} 53$ mutations}

To investigate the incidence of TP53 mutations in classic ILC and PILC, we performed PCR on exons 4-9 (conserved midregion) of TP53 for 41 ILC cases (22 classic ILC and 19 PILC). Direct DNA sequencing was subsequently performed on PCR products. Overall, we detected 11 mutations (of which 1 novel and 10 previously reported) and 2 validated polymorphisms in 41 ILC cases (Tables 2 and 3). One out of 11 mutations was located in an intron and 10 mutations were located in coding regions. Using the freely available IARC TP53 database, we have scrutinized the following; the functions of the domains in which the mutated residues are located, the known functions of the wild-type residues, the effect of the mutations, the predicted effect on splicing, functional predictions based on the structure change and previously reported tumor sites (Table 2) $[18,19]$. This data summarized in Table 2 allowed us to predict the pathogenicity of the observed mutations. We conclude that all of the 11 mutations found could potentially be pathogenic based on the mentioned criteria above.

Next, we evaluated the distribution of these potentially pathogenic mutations over classic and pleomorphic ILC variants. Eight of the 19 PILC cases (42.1\%) exhibited a potentially pathogenic mutation which is significantly more often when compared to the percentage of potentially pathogenic mutations found in classic ILC cases ( 3 mutations (missense) observed in 22 classic ILC cases $(13.6 \% ; p \leq 0.05)$ ) (Table 4$)$. We have also observed two previously reported and validated polymorphisms among our samples. These were distributed similarly over the classic and pleomorphic ILC variants (Table 3).

\section{$3.2 \mathrm{p} 53$ expression and accumulation}

p53 immunostaining was performed to investigate the correlation of mutational status and effects on protein expression (Fig. 1). Immunohistochemistry scores and mutation data of each case are summarized in Table 2 . We observed a variation in the immunohistochemistry scores of p53 in both pleomorphic and classic cases. In normal breast tissue, p53 staining is observed in a small percentage (approximately 1\%) of the cells. Therefore, $5 \%$ positivity was chosen as a value to distinguish normal, wild-type p53 expression and mutated p53 overexpression. Overall, 9/41 cases $(21.9 \%)$ showed p53 accumulation, while 6 cases showed absence of expression. Of these 15 cases, 6 were ILC and 9 PILC ( $p=0.157)$. In conclusion, p53 accumulation was not associated exclusively with a specific ILC variant.

\subsection{Correlation of $\mathrm{p} 53$ accumulation and mutation}

Because a large body of literature has shown that p53 accumulation can be caused by mutations in TP53, we set out to investigate the correlation between p53 accumulation and mutation. In total, 5 out of 9 cases that showed p53 accumulation based on immunohistochemistry harbored mutations (Table 5). Interestingly, we detected potential pathogenic mutations in 4 of the 32 cases without $\mathrm{p} 53$ accumulation (wild-type staining $+0 \%$ staining) $(p=0.01)$, while 1 of 6 cases showed a pathogenic mutation in the absence of $\mathrm{p} 53$ staining $(0 \%$ staining). However, the 5 remaining cases with $0 \%$ staining had one of the two validated polymorphisms (Number 2 in Table 3). In summary, we observed a significant correlation of p53 accumulation with potentially pathogenic mutations (Table 5). 


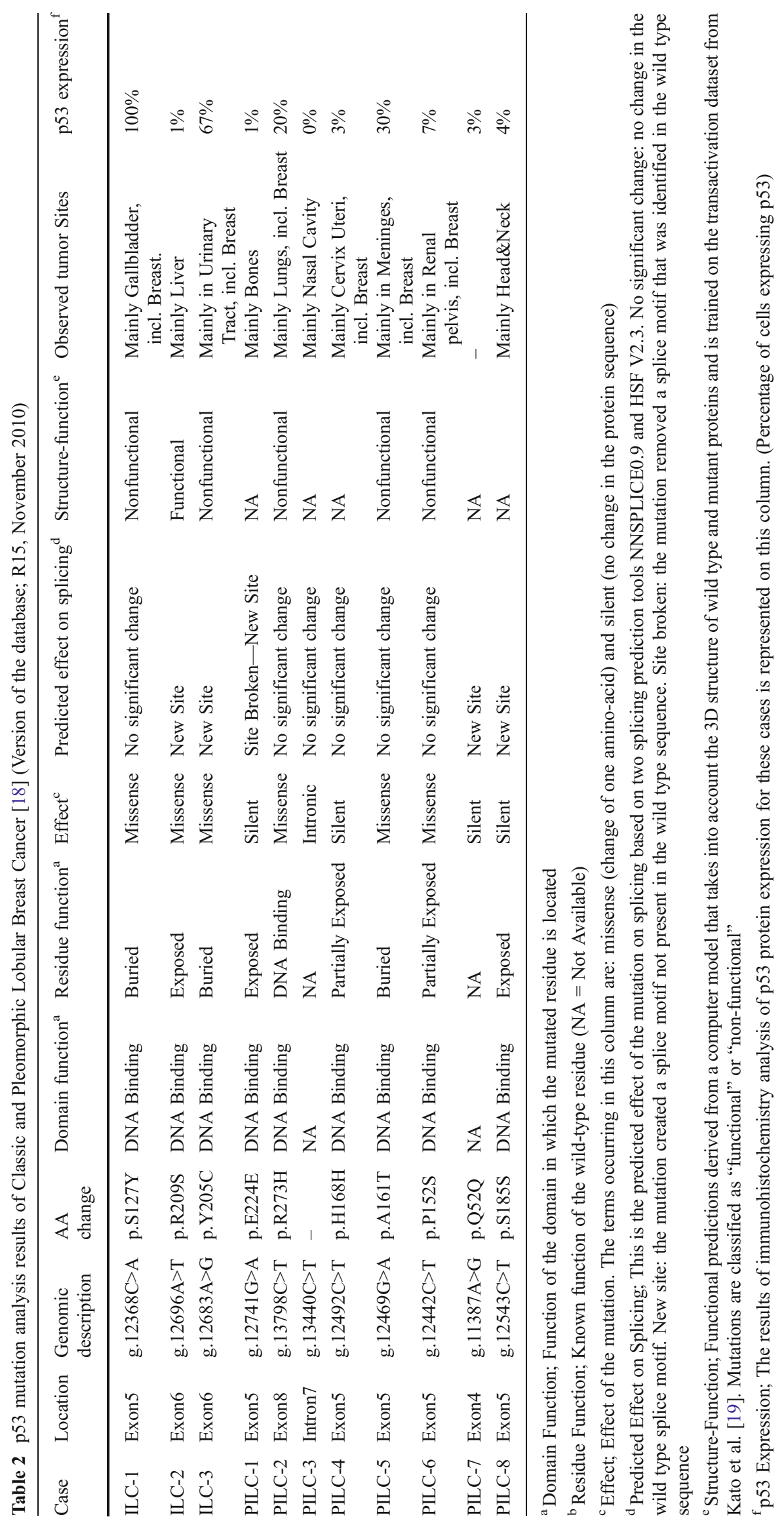


Table 3 p53 mutation analysis results of Classic and Pleomorphic Lobular Breast Cancer [18] (Version of the database; R15, November 2010)

\begin{tabular}{llllllll}
\hline Number & Location & $\begin{array}{l}\text { Genomic } \\
\text { description }\end{array}$ & $\begin{array}{l}\text { Aminoacid } \\
\text { change }\end{array}$ & $\begin{array}{l}\text { Domain } \\
\text { function }^{\mathrm{b}}\end{array}$ & Effect $^{\mathrm{c}}$ & $\begin{array}{l}\text { Predicted effect } \\
\text { on splicing }^{\mathrm{d}}\end{array}$ & Observed in \\
\hline 1 & Exon4 & g.11339G $>\mathrm{A}$ & $36 \mathrm{P}>\mathrm{P}$ & Transactivation & Silent & NA & 3 PILC and 3 ILC cases \\
2 & Exon4 & g.11446C $>\mathrm{G}^{\mathrm{a}}$ & $\begin{array}{l}72 \mathrm{P}>\mathrm{R} \\
\text { SH3-like/Pro-rich }\end{array}$ & Missense & New Site & 11 PILC and 18 ILC cases \\
\hline
\end{tabular}

${ }^{a}$ Several studies showed its association with breast cancer in different populations

${ }^{\mathrm{b}}$ Domain Function; Function of the domain in which the mutated residue is located

${ }^{\mathrm{c}}$ Effect; Effect of the mutation. The terms occurring in this column are: missense (change of one amino-acid) and silent (no change in the protein sequence)

${ }^{\mathrm{d}}$ Predicted Effect on Splicing; This is the predicted effect of the mutation on splicing based on two splicing prediction tools NNSPLICE0.9 and HSF V2.3. No significant change: no change in the wild type splice motif. New site: the mutation created a splice motif which is not present in the wt sequence. Site broken: the mutation removed a splice motif that was identified in the wt sequence. $(\mathrm{NA}=$ Not available)

\section{Discussion}

Pleomorphic ILC was first described in 1982 as a variant of infiltrating lobular carcinoma [20]. Even though the morphology of PILC with high nuclear atypia is distinctive, its feature of high frequency of multicentrity and bilaterality seem to be similar to the classic ILC [21]. The report of Weidner and Semple [9] is one of several reports demonstrating the aggressive clinical behavior and poorer prognosis of patients with PILC in comparison to patients with ILC [9]. Therefore, despite the similarities between them, the question mark on the genetic pathways through which each variant evolves still remains. The TP53 tumor suppressor gene has been an interesting target to investigate in invasive breast cancer since it is very frequently altered in other human cancers [22]. Many research groups have investigated the distribution of p53 mutations and its correlation with immunohistochemistry in invasive carcinomas [23-27] but data focusing on different variants of ILC are limited. Therefore our aim was to study the mutational status of p53 in classic and pleomorphic ILC to gain a better understanding of the molecular changes occurring in this gene, which possibly contribute to the development of these subtypes and the potential of it as a tool to differentially diagnose ILC versus PILC.

In the present work, we studied 41 ILC cases for p53 mutations and accumulation in relation to the classic and pleomorphic variants. Eleven mutations were detected in 41

Table 4 Correlation of potentially pathogenic p53 mutations with classic and pleomorphic invasive lobular carcinomas

\begin{tabular}{llll}
\hline & \multicolumn{2}{l}{ Potentially pathogenic p53 mutations } & \\
\cline { 2 - 3 } & + & - & \\
\hline Classic ILC & $3(13.6 \%)$ & $19(86.4 \%)$ & Total 22 \\
PILC & $8(42.1 \%)$ & $11(57.9 \%)$ & Total 19 \\
\hline
\end{tabular}

$p<0.05 *$ cases studied (26\%) which is in line with the literature which states that the overall frequency of p53 mutations in breast cancer is approximately 20\% [28]. Almost all the observed mutations locate in the highly conserved DNA-binding domain of the protein [18] (Table 2). Interestingly, our mutation analysis reveals that PILC is associated with a higher frequency (42.1\%) of potentially pathogenic p53 mutations compared to ILC (13.6\%). Even though some of these potentially pathogenic mutations (4 out of 11 mutations) do not result in an amino acid change, they have been reported before in different solid tumors including breast cancer [18]. These silent mutations are of particular interest. It has already been known for decades that non-transforming mutations can affect the protein production and therefore the function by interfering with various phases of transcription and translation [29]. Examples to possible scenarios are: i) interference with the editing of a gene transcript if silent mutations occur in codons that contain splicing enhancers responsible for the proper removal of introns, or ii) interference with the stability of mRNA by preventing correct folding; thus affecting the speed of translation of the protein as well as the degradation of the mRNA. Strikingly, Lamolle et al. recently showed that all reported nontransforming mutations of p53 which are documented in p53 somatic mutation database are preferentially located in conserved amino acid positions, which may depict their importance [30]. The majority of these mutations were found as single mutations, so never associated with other mutations in p53 gene, and they tended to be located inside suspected splicing enhancers. Interestingly, silent mutations observed in our study also exist as single mutations and locate either in an exposed residue of the DNA-binding domain or cause a predicted change in splicing. Moreover, silent mutations in p53 may also lead to a functional response by interfering with binding to MDM2. In this scenario, single silent point mutations in p53 mRNA can disrupt its binding to MDM2 resulting in aberrant p53 synthesis and degradation [31]. This failure in binding is shown to reduce $\mathrm{p} 53$ activity, thereby supporting the notion that silent p53 mutations can affect p53 function. 
Fig. 1 p53 expression in PILC. a-d. Representative examples of 553 expression of PILC cases (a) wild-type; $1 \%$ positive nuclei (b) no expression and absence of mutations (c) 'g. $12368 \mathrm{C}>\mathrm{A}$ ' mutation resulting in $100 \%$ p 53 overexpression (d) ' $\mathrm{g} .13440 \mathrm{C}>\mathrm{T}$ ' mutation occurring in an intron site and resulting in absence of p53 expression a)

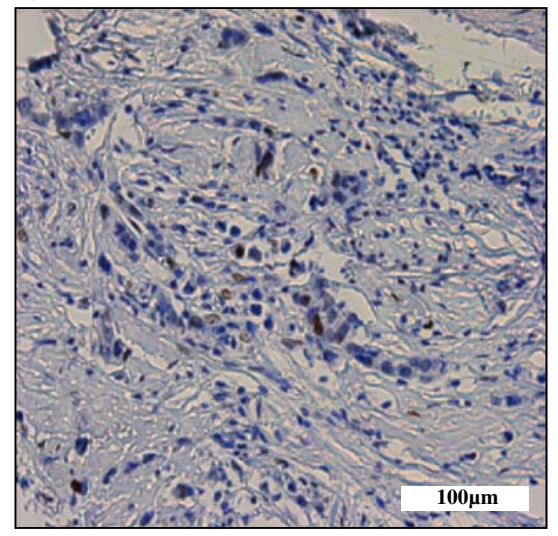

c)

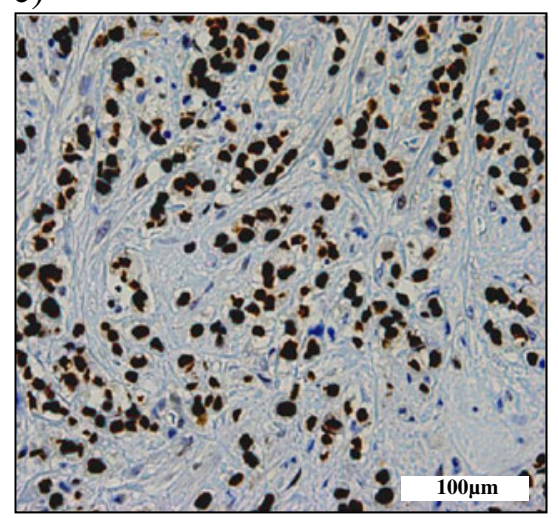

b)

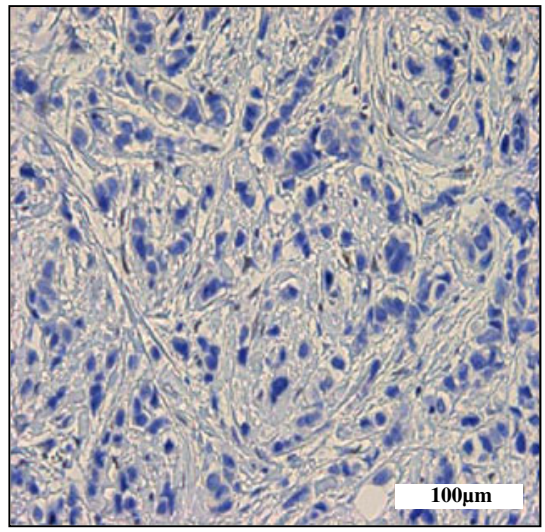

d)

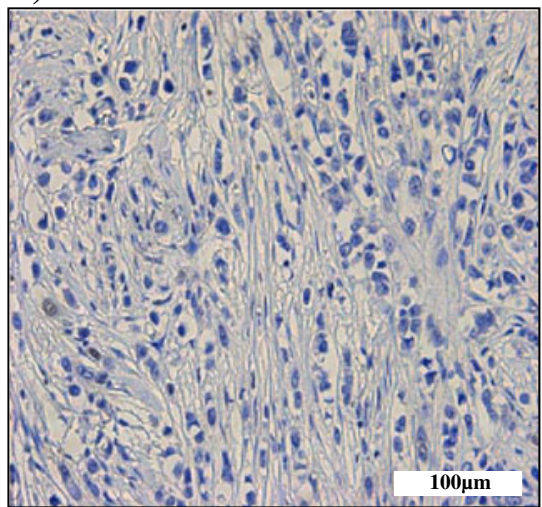

Therefore, we think that our observation is noteworthy and in the light of recent discoveries in this field, assumptions on nontransforming mutations should be made carefully. We have also observed two previously reported and validated polymorphisms in a high number of our cases with no significant preference for either ILC variant. Of note is the fact that these polymorphisms were shown to be related with cancer susceptibility [18]. Especially the amino acid change $72 \mathrm{P}>\mathrm{R}$ (Table 3 ) has been shown to be associated with breast cancer susceptibility [32-36].

The observation of this significantly increased frequency of pathogenic mutations in PILC -more than half of which do probably give rise to a non-functional protein based on

Table 5 Correlation between p53 mutations that give rise to a nonfunctional structure and p53 accumulation

Mutations causing a predicted defect in $\mathrm{p} 53$ structure

\begin{tabular}{llll}
\hline $\mathrm{p} 53$ & -32 & 0 \\
Accumulation & + & 4 & 5 \\
\hline
\end{tabular}

$p \leq 0.001^{*}$ predictions of computer models and for some based on literature (Table 2)- is also in line with mouse studies in which stochastic somatic inactivation of $\mathrm{p} 53$ and E-cadherin in the mammary gland induced the development of mouse PILC [16]. For some of the observed potentially pathogenic mutations, predictions on function of the protein were not available. However, it would not be unexpected if there were activating mutations among them since mutant p53 protein can also have a distinct function in cancer such as promoting invasion and metastases [37].

Another observation in this study was the significant correlation between p53 accumulation and transforming pathogenic mutations independent of any ILC variant. The positive correlation between p53 accumulation and mutations were made by other groups $[4,38-40]$ although not by all [41, 42]. It is apparent that the correlation between accumulation and mutations also depends on the influence of the mutation on the half-life of the protein. Most mutant forms of $\mathrm{p} 53$ have a longer cellular life and are therefore recognized by antibodies while others are not. This can explain our differential observation of p53 accumulation when all mutations are counted. A mutation might cause a misfolding of the protein structure (such as the above mentioned silent mutations resting in splicing enhancers or playing a role in mRNA stability) or a truncation that affects the 
epitope that is recognized by the antibody. We have also observed a case with p53 accumulation but no mutations. An explanation for this may be the possibility of mutations that are located outside of the investigated exons. Interestingly, a high percentage of p53-negative cases showed a polymorphism in residue 72 , a mutation that has been implicated in enhanced targeting for degradation [43] providing a possible explanation for the p53 negativity in these cases. However, based on published data and our results, we can conclude that p53 immunostaining does not always reflect the genetic alteration and vice versa; the existence of a mutation does not always lead to p53 accumulation or complete lack of expression.

In conclusion, the clinically more aggressive pleomorphic variant of ILC bears a significantly higher frequency of p53 mutations compared to the classic variant, Moreover, since inactivation of $\mathrm{p} 53$ and E-cadherin in mice leads to the development of PILC, we envisage that p53 mutations may play a role in carcinogenesis of PILC.

Acknowledgments We thank Dr. Petra van der Groep for advice on immunohistochemistry.

Conflict of interest The authors declare no conflict of interests.

Open Access This article is distributed under the terms of the Creative Commons Attribution License which permits any use, distribution, and reproduction in any medium, provided the original author(s) and the source are credited.

\section{References}

1. C.C. Harris, Structure and function of the $\mathrm{p} 53$ tumor suppressor gene: clues for rational cancer therapeutic strategies. J. Natl. Cancer Inst. 88, 1442-1455 (1996)

2. M. Gasco, S. Shami, T. Crook, The p53 pathway in breast cancer. Breast Cancer Res. 4, 70-76 (2002)

3. B.J. Chae, J.S. Bae, A. Lee, W.C. Park, Y.J. Seo, B.J. Song, J.S. Kim, S.S. Jung, p53 as a specific prognostic factor in triple-negative breast cancer. Jpn. J. Clin. Oncol. 39, 217-224 (2009)

4. P. Rossner Jr., M.D. Gammon, Y.J. Zhang, M.B. Terry, H. Hibshoosh, L. Memeo, M. Mansukhani, C.M. Long, G. Garbowski, M. Agrawal, T.S. Kalra, M.M. Gaudet, S.L. Teitelbaum, A.I. Neugut, R.M. Santella, Mutations in p53, p53 protein overexpression and breast cancer survival. J. Cell Mol. Med. 13, 3847-3857 (2009)

5. E. Gudlaugsson, I. Skaland, E.A. Janssen, P.J. van Diest, F.J. Voorhorst, K. Kjellevold, A. zur Hausen, J.P. Baak, Prospective multicenter comparison of proliferation and other prognostic factors in lymph node negative lobular invasive breast cancer. Breast Cancer Res. Treat. 121, 35-40 (2010)

6. N. Perry, M. Broeders, C. de Wolf, S. Tornberg, R. Holland, L. von Karsa, European guidelines for quality assurance in breast cancer screening and diagnosis. Fourth edition-summary document. Ann. Oncol. 19, 614-622 (2008)
7. H.P.R. Kini, A.R. Rau, F.D. Lobo, A.J. Augustine, B.S. Ramesh, Pleomorphic lobular carcinoma of the breast-a diagnostic dilemma. J. Cytol. 24, 193-195 (2007)

8. V. Eusebi, F. Magalhaes, J.G. Azzopardi, Pleomorphic lobular carcinoma of the breast: an aggressive tumor showing apocrine differentiation. Hum. Pathol. 23, 655-662 (1992)

9. N. Weidner, J.P. Semple, Pleomorphic variant of invasive lobular carcinoma of the breast. Hum. Pathol. 23, 1167-1171 (1992)

10. J.S. Bentz, N. Yassa, F. Clayton, Pleomorphic lobular carcinoma of the breast: clinicopathologic features of 12 cases. Mod. Pathol. 11, 814-822 (1998)

11. L.P. Middleton, D.M. Palacios, B.R. Bryant, P. Krebs, C.N. Otis, M.J. Merino, Pleomorphic lobular carcinoma: morphology, immunohistochemistry, and molecular analysis. Am. J. Surg. Pathol. 24, 1650-1656 (2000)

12. D. Frolik, R. Caduff, Z. Varga, Pleomorphic lobular carcinoma of the breast: its cell kinetics, expression of oncogenes and tumour suppressor genes compared with invasive ductal carcinomas and classical infiltrating lobular carcinomas. Histopathology 39, 503513 (2001)

13. N. Sneige, J. Wang, B.A. Baker, S. Krishnamurthy, L.P. Middleton, Clinical, histopathologic, and biologic features of pleomorphic lobular (ductal-lobular) carcinoma in situ of the breast: a report of 24 cases. Mod. Pathol. 15, 1044-1050 (2002)

14. G. Mazoujian, C. Bodian, D.E. Haagensen Jr., C.D. Haagensen, Expression of GCDFP-15 in breast carcinomas. Relationship to pathologic and clinical factors. Cancer 63, 2156-2161 (1989)

15. P.W. Derksen, X. Liu, F. Saridin, H. van der Gulden, J. Zevenhoven, B. Evers, J.R. van Beijnum, A.W. Griffioen, J. Vink, P. Krimpenfort, J.L. Peterse, R.D. Cardiff, A. Berns, J. Jonkers, Somatic inactivation of E-cadherin and p53 in mice leads to metastatic lobular mammary carcinoma through induction of anoikis resistance and angiogenesis. Cancer Cell 10, 437-449 (2006)

16. P.W. Derksen, T.M. Braumuller, E. van der Burg, M. Hornsveld, E. Mesman, J. Wesseling, P. Krimpenfort, J. Jonkers, Mammaryspecific inactivation of E-cadherin and p53 impairs functional gland development and leads to pleomorphic invasive lobular carcinoma in mice. Dis. Model Mech. 3, 347-358 (2011)

17. P.J. van Diest, No consent should be needed for using leftover body material for scientific purposes. For. Bmj 325, 648-651 (2002)

18. A. Petitjean, E. Mathe, S. Kato, C. Ishioka, S.V. Tavtigian, P. Hainaut, M. Olivier, Impact of mutant p53 functional properties on TP53 mutation patterns and tumor phenotype: lessons from recent developments in the IARC TP53 database. Hum. Mutat. 28, 622-629 (2007)

19. S. Kato, S.Y. Han, W. Liu, K. Otsuka, H. Shibata, R. Kanamaru, C. Ishioka, Understanding the function-structure and functionmutation relationships of p53 tumor suppressor protein by highresolution missense mutation analysis. Proc. Natl. Acad. Sci. U. S. A. 100, 8424-8429 (2003)

20. J.M. Dixon, T.J. Anderson, D.L. Page, D. Lee, S.W. Duffy, Infiltrating lobular carcinoma of the breast. Histopathology 6, 149-161 (1982)

21. D.L. Page, T.J. Anderson, Diagnostic histopathology of the breast. (1987)

22. M. Hollstein, D. Sidransky, B. Vogelstein, C.C. Harris, p53 mutations in human cancers. Science 253, 49-53 (1991)

23. J. Bartek, J. Bartkova, B. Vojtesek, Z. Staskova, A. Rejthar, J. Kovarik, D.P. Lane, Patterns of expression of the p53 tumour suppressor in human breast tissues and tumours in situ and in vitro. Int. J. Cancer 46, 839-844 (1990)

24. I.B. Runnebaum, M. Nagarajan, M. Bowman, D. Soto, S. Sukumar, Mutations in p53 as potential molecular markers for human breast cancer. Proc. Natl. Acad. Sci. U. S. A. 88, 10657-10661 (1991) 
25. A.M. Thompson, T.J. Anderson, A. Condie, J. Prosser, U. Chetty, D.C. Carter, H.J. Evans, C.M. Steel, p53 allele losses, mutations and expression in breast cancer and their relationship to clinico-pathological parameters. Int. J. Cancer 50, 528-532 (1992)

26. A.D. Thor, D.H. Moore II, S.M. Edgerton, E.S. Kawasaki, E. Reihsaus, H.T. Lynch, J.N. Marcus, L. Schwartz, L.C. Chen, B. H. Mayall et al., Accumulation of p53 tumor suppressor gene protein: an independent marker of prognosis in breast cancers. J. Natl. Cancer Inst. 84, 845-855 (1992)

27. T.I. Andersen, R. Holm, J.M. Nesland, K.R. Heimdal, L. Ottestad, A.L. Borresen, Prognostic significance of TP53 alterations in breast carcinoma. Br. J. Cancer 68, 540-548 (1993)

28. P.D. Pharoah, N.E. Day, C. Caldas, Somatic mutations in the p53 gene and prognosis in breast cancer: a meta-analysis. Br. J. Cancer 80, 1968-1973 (1999)

29. J.V. Chamary, L.D. Hurst, The price of silent mutations. Sci. Am. 300, 46-53 (2009)

30. G. Lamolle, M. Marin, F. Alvarez-Valin, Silent mutations in the gene encoding the $\mathrm{p} 53$ protein are preferentially located in conserved amino acid positions and splicing enhancers. Mutat. Res. 600, 102-112 (2006)

31. M.M. Candeias, L. Malbert-Colas, D.J. Powell, C. Daskalogianni, M.M. Maslon, N. Naski, K. Bourougaa, F. Calvo, R. Fahraeus, P53 mRNA controls p53 activity by managing Mdm2 functions. Nat. Cell Biol. 10, 1098-1105 (2008)

32. N. Buyru, H. Tigli, N. Dalay, P53 codon 72 polymorphism in breast cancer. Oncol. Rep. 10, 711-714 (2003)

33. T. Ohayon, R. Gershoni-Baruch, M.Z. Papa, T. Distelman Menachem, S. Eisenberg Barzilai, E. Friedman, The R72P P53 mutation is associated with familial breast cancer in Jewish women. Br. J. Cancer 92, 1144-1148 (2005)

34. C. Noma, Y. Miyoshi, T. Taguchi, Y. Tamaki, S. Noguchi, Association of p53 genetic polymorphism (Arg72Pro) with estrogen receptor positive breast cancer risk in Japanese women. Cancer Lett. 210, 197-203 (2004)
35. M.M. Siddique, C. Balram, L. Fiszer-Maliszewska, A. Aggarwal, A. Tan, P. Tan, K.C. Soo, K. Sabapathy, Evidence for selective expression of the 553 codon 72 polymorphs: implications in cancer development. Cancer Epidemiol. Biomarkers Prev. 14, 2245-2252 (2005)

36. T.G. Kalemi, A.F. Lambropoulos, M. Gueorguiev, S. Chrisafi, K.T. Papazisis, A. Kotsis, The association of p53 mutations and p53 codon 72, Her 2 codon 655 and MTHFR C677T polymorphisms with breast cancer in Northern Greece. Cancer Lett. 222, 57-65 (2005)

37. P.A. Muller, P.T. Caswell, B. Doyle, M.P. Iwanicki, E.H. Tan, S. Karim, N. Lukashchuk, D.A. Gillespie, R.L. Ludwig, P. Gosselin, A. Cromer, J.S. Brugge, O.J. Sansom, J.C. Norman, K.H. Vousden, Mutant $\mathrm{p} 53$ drives invasion by promoting integrin recycling. Cell 139, 1327-1341 (2009)

38. J. Lukas, N. Niu, M.F. Press, p53 mutations and expression in breast carcinoma in situ. Am. J. Pathol. 156, 183-191 (2000)

39. F.C. Schmitt, R. Soares, L. Cirnes, R. Seruca, P53 in breast carcinomas: association between presence of mutation and immunohistochemical expression using a semiquantitative approach. Pathol. Res. Pract. 194, 815-819 (1998)

40. Y. Umekita, K. Kobayashi, T. Saheki, H. Yoshida, Nuclear accumulation of $\mathrm{p} 53$ protein correlates with mutations in the p53 gene on archival paraffin-embedded tissues of human breast cancer. Jpn. J. Cancer Res. 85, 825-830 (1994)

41. B.W. Lisboa, S. Vogtlander, T. Gilster, L. Riethdorf, K. MildeLangosch, T. Loning, Molecular and immunohistochemical analysis of p53 mutations in scrapings and tissue from preinvasive and invasive breast cancer. Virchows Arch. 431, 375-381 (1997)

42. D. Lohmann, C. Ruhri, M. Schmitt, H. Graeff, H. Hofler, Accumulation of p53 protein as an indicator for p53 gene mutation in breast cancer. Occurrence of false-positives and false-negatives. Diagn. Mol. Pathol. 2, 36-41 (1993)

43. A. Storey, M. Thomas, A. Kalita, C. Harwood, D. Gardiol, F. Mantovani, J. Breuer, I.M. Leigh, G. Matlashewski, L. Banks, Role of a p53 polymorphism in the development of human papillomavirus-associated cancer. Nature 393, 229-234 (1998) 